NOTE TECHNIQUE

\title{
AMÉLIORATION DES CONDITIONS DE CAPTURE PRINTANIÈRE ET DE STABULATION DE SAUMONS ADULTES POUR LA REPRODUCTION ARTIFICIELLE
}

\author{
R. CUINAT *, P. BOMASSI * et A. CARRIER **
}

PLAN

Résumé.

Abstract.

1 - Introduction.

II - Bref rappel de l'écologie du Saumon Loire-Allier.

III - Le problème de l'approvisionnement en œufs pour le repeuplement, et notamment de la stabulation des reproducteurs.

A - Choix de la meilleure stratégie possible pour le repeuplement en saumons dans l'Allier.

B - Difficultè à stabuler longtemps des saumons adultes.

(*) Délégué Régional.

(**) Chargé d'Etudes.

$\left({ }^{* *}\right)$ Garde-Pêche Commissionné de l'Administration $6^{\mathrm{r}}$ Délégation Régionale du Conseil Supérieur de la Pêche. 84, avenue du Puy-de-Dôme 63000 CLERMONT-FERRAND. 
IV - Techniques de capture.

A - Caractéristiques essentielles du barrage de Vichy. $\quad 129$

B - Captures dans l'échelle Lachadenède. $\quad 130$

C - Captures dans le parcours kayak. 133

D - Périodes et conditions de piégeage. 133

V - Transport et déversement à la pisciculture. $\quad 134$

VI - Stabulation jusqu'à maturité sexuelle. Résultats. 136

$\begin{array}{ll}\text { A - Bassins de dimensions moyennes à la salmoniculture } & \\ \text { du Conseil Supérieur de la Pêche à Augerolles. } & 136\end{array}$

$B$ - Bassin de grande dimension, à Theix. 136

$\begin{array}{ll}\text { C - Bassin de grande dimension, à Augerolles. } & 137\end{array}$

D - Tri des reproducteurs et ponte artificielle. 138

VII - Discussion et conclusions. $\quad 140$

\section{RESUME}

Le pourcentage de Saumons parvenant jusqu'à leurs meilleures zones de frayeres sur le Haut-Allier est souvent faible, du fait de la longueur du parcours $(800$ à $860 \mathrm{~km})$ et des nombreux obstacles. Pour ne pas affecter directement ce potentiel de reproduction naturelle, les sujets destinés à la ponte artificielle sont prélevés loin en aval et longtemps avant la saison de reproduction, c'est-à-dire au barrage de Vichy (03) en avril et plus récemment en mai.

Malheureusement, les mortalitès sur les Saumons en captivité étaient très élevèes (surtout dans les six semaines suivant la capture) lors des premières campagnes (1970 à 1973) : 80 à $100 \%$. Elles ont pu être progressivement ré-; duites dans les campagnes suivantes et sont descendues à $15 \%$ en 1978 (34 sujets capturés) et $7 \%$ en 1979 (44 sujets).

Cette amélioration est lièe essentiellement :

a) au perfectionnement du matériel et des techniques de piégeage, manipulation et transport des Saumons, de façon à limiter au maximum fatigue, traumatisme ou stress :

b) à la stabulation dans un bassin de très grandes surface $\left(8000 \mathrm{~m}^{2}\right)$ et profondeur (moyenne : $2 \mathrm{~m}$, maximum : $4 \mathrm{~m}$ ), avec fort débit (environ $100 \mathrm{l} / \mathrm{sec}$ ), où l'eau ne dépasse pas $18^{\circ} \mathrm{C}$.

Divers perfectionnements sont encore nécessaires. Mais le fait de mieux maitriser l'approvisionnement en œufs permet maintenant d'envisager une intensification des repeuplements sur ce bassin, en réduisant ou supprimant les importations d'œufs (Grande-Bretagne ou Scandinavie); nos marquages ont, en effet, montré que les retours d'adultes étaient deux à trois fois supérieurs à partir d'œufs de souche Allier. 


\section{ABSTRACT}

\section{IMPROVEMENT OF SPRING CAPTURE AND HOLDING CONDITIONS OF ADULTS SALMON FOR ARTIFICIAL REPRODUCTION}

The percentage of Salmon joining their best reproduction areas, in the upper part of Allier River, is often small because of the length of the course $(800$ to $860 \mathrm{~km})$ and the number of obstacles. So, to preserve this natural potential of repro. duction, adults needed for artificial reproduction are removed below these areas a long time before spawning, namely at Vichy dam (03) in April and more recently in May.

Unfortunately, mortalities among captive Salmon were very important (especially during the six weeks following capture) during the first trials (1970 to 1973 ) : 80 to $100 \%$. They were gradually reduced in the following campaigns and reached $15 \%$ in 1978 (34 adults collected) and $7 \%$ in 1979 (44 aduits)

This amelioration is mainly connected with :

a) the progress in equipment and in technics of capture, handling and transport of Salmon, to minimise fatigue, injury or stress ;

b) holding in very large $\left(8000 \mathrm{~m}^{2}\right)$ and deep (2 to $\left.4 \mathrm{~m}\right)$ pond, in which water inflow is about $100 \mathrm{l} / \mathrm{sec}$ and maximum water temperature is $18^{\circ} \mathrm{C}$.

Different improvements are still necessary. But, because we are now able to secure regular supply of eggs, it will be possible to increase stocking for this river system, without help of imported eggs (from U.K. or Scandinavia); our taggings showed that returns as adults are two or three times higher when planted smolts are from native eggs.

\section{INTRODUCTION}

La survie du saumon atlantique (Salmo salar L.) devient de plus en plus difficile sur l'axe Loire-Allier, du fait surtout de la multiplication ou de l'aggravation des barrages et des nouveaux obstacles indirectement provoqués par les extractions intensives de granulats dans le lit de ces deux cours d'eau.

Pour tenter de soutenir - et si possible d'augmenter - ses effectifs, des mesures de plusieurs types ont été prises, principalement :

- depuis 1974 surtout, réglementation tendant à réduire les risques de sur-exploitation;

- amélioration des repeuplements. en qualité et en quantité.

Pour ces repeuplements, le Conseil Supérieur de la Pêche a créé, en 1968, une salmoniculture consacrèe presque entièrement à l'élevage de jeunes saumons jusqu'au stade saumoneau (ou "smolt"). Ses installations, de type semi-extensif, permettent de produire des sujets relativement rustiques.

Le plus gros problème a èté, depuis le démarrage de cet établissement, celui de son approvisionnement régulier en œufs de qualité.

Le chapitre II résume brièvement l'écologie du saumon d'ans ce bassin, qui conditionne la * stratégie * qui nous sembie la plus adaptée en matière de 
capture de reproducteurs (chapitre III). Les chapitres suivants decrivent les diverses pratiques qui ont permis au Conseil Supérieur de la Pêche de résoudre de mieux en mieux les problèmes de stabulation de ces saumons géniteurs.

\section{BREF RAPPEL DE L'ECOLOGIE SAUMON LOIRE-ALLIER}

Le Saumon Atlantique (Salmo salar L.) du bassin de la Loire ne se reproduit plus, depuis plusieurs décennies, que dans son affluent principal, l'Allier. Les meilleures frayères se situent dans la partie supérieure de cette rivière, entre Brioude et le barrage infranchissable de Poutès, c'est-à-dire à $800-860 \mathrm{~km}$ de l'estuaire de la Loire.

Du fait probablement d'une adaptation à cette distance très importante, les saumons de ce bassin fluvial se présentent très tôt en estuaire : de novembre jusqu'au printemps, c'est-à-dire 12 à 6 mois avant la période de ponte : minovembre à mi-décembre. Mais la multiplication des obstacles, en Loire et dans l'Allier, rend la montée de plus en plus lente et aléatoire. Il est maintenant fréquent que des saumons se trouvent encore en aval de Vichy au début de mai. Leurs chances de parvenir jusqu'à la zone des bonnes frayères sont alors réduites, car les debits vont rapidement diminuer et les températures augmenter, alors qu'il reste six obstacles à franchir à l'amont de cette ville.

La pêche est pratiquée, en estuaire et en Loire, essentiellement au filet, par des professionnels; dans l'Allier, les saumons ne sont pêchés qu'à la ligne. Les dates d'ouverture de la pêche ont été retardèes d'environ un mois, depuis 1974, afin d'épargner surtout la fraction * précoce» du contingent ( ${ }^{*}$ ). Les nombres de saumons capturés ont diminué de plus de 95 pour cent depuis le début du siècle et semblent varier, dans la dernière décennie, entre 500 et 3000 selon les annèes $\left({ }^{* *}\right)$

Les juvéniles ont une croissance très rapide dans l'Allier; la plus grande partie (80 à $90 \%$ selon les années) se smoltifie dès l'âge de 13-15 mois et émigre vers la mer en avril et mai. Les marquages ont montré que ces sujets gagnaient la façade Ouest du Groënland. Le retour en Loire s'effectue génèralement après deux ètés $(47 \%$ ) ou trois ètés $(51 \%)$ de grossissement en mer.

(*) Dates actuelles approximatives d'ouverture de la pêche au saumon:

- en estuaire (aval de Nantes) : 11 janvier:

- en Loire : 3 février dans la partie aval, 5 mars partie amont:

- dans l'Allier : 5 mars, partie aval, 12 mars sur le reste de la rivière.

Fermeture : 15 juin.

(**) Les captures en Loire, et surtout en estuaire, sont très mal connues; elles constituent peut-être de 40 à 60 pour cent des captures totales en eau douce. 


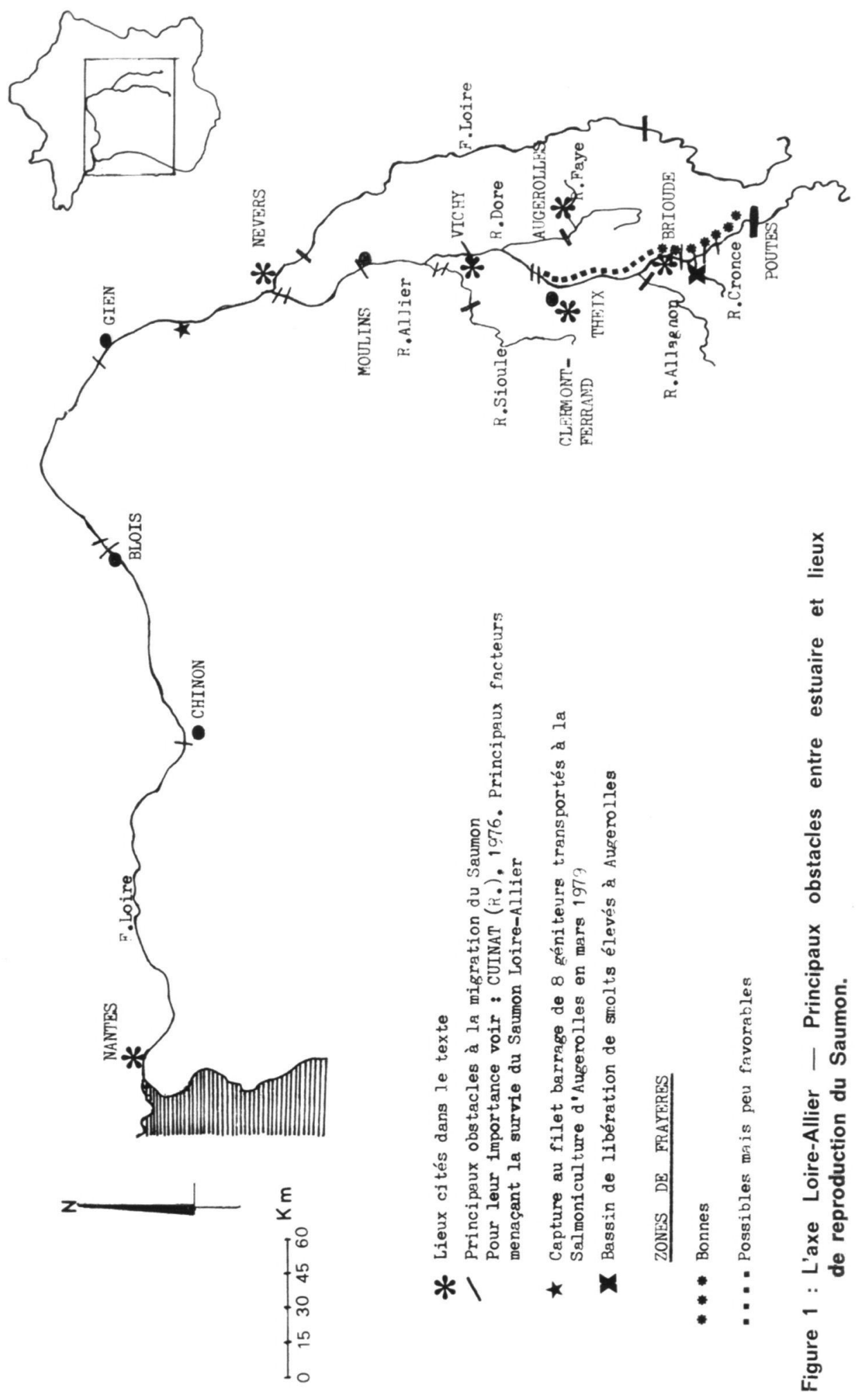




\section{LE PROBLEME DE L'APPROVISIONNEMENT EN GEUFS POUR LE REPEU. PLEMENT, ET NOTAMMENT DE LA STABULATION DES REPRODUCTEURS}

\section{A. Choix de la meilleure stratégie possible pour le repeuplement on saumons dans l'Allier} d'œufs :

Des repeuplements ont été pratiqués dans l'Allier de longue date, à partir

- soit importés (de Grande-Bretagne ou de Scandinavie principalement) :

- soit indigènes.

Les marquages ayant montré que les seconds donnaient lieu à des taux de retours en rivière deux à trois fois plus élevés, il importait de disposer avant tout d'œufs de saumons Loire-Allier, les œufs importés n'étant à considérer que comme un appoint éventuel.

Avant 1973, les saumons reproducteurs ont presque toujours été capturés sur le Haut-Allier :

- soit dans l'échelle à poissons du barrage de Brioude,

- soit à la pêche électrique (ou exceptionnellement même à la ligne), sur les frayères ou à proximité immédiate.

Dans les deux cas. il s'agissait de sujets ayant déjà surmonté la plupart des obstacles et ayant survécu jusqu'à la période de ponte. Le prélèvement s'effectuait donc au détriment direct $d u$ potentiel de reproduction naturelle, ce qui était biologiquement très critiquable, étant donné que :

- les frayères du Haut-Allier sont maintenant très loin d'être saturées en reproducteurs: l'espace disponible pour les œufs, alevins, tacons, est très important, à l'amont de Brioude (au moins 30 hectares de frayères et 100 hectares de zone convenant aux tacons);

- il n'est pas certain que l'élevage à la salmoniculture d'Augerolles donne des résultats très supérieurs à la reproduction naturelle dans cette partie supérieure de l'Allier, en terme de rendement global (nombre de retours d'adultes à partir d'un nombre donné d'œufs pondus).

II nous est donc apparu biologiquement bien préférable que les saumons destinés à la reproduction artificielle soient prélevés le plus en aval possible. de facon à n'affecter que très peu la reproduction naturelle 11 est intéressant également de s'adresser plutôt à des contingents " retardés", dont les chances de parvenir jusqu'à la zone des bonnes frayères sont réduites.

Cette option s'est cependant heurtèe à la difficulté de conserver jusqu'à maturité les saumons prélevés longtemps avant maturité sexuelle.

\section{B. Difficulté à stabuler longtemps des saumons adultes}

Bien que le problème de leur nourrissage ne se pose pas, puisqu'ils ne s'alimentent pas en eau douce, les saumons adultes sont difficiles à conserver en captivité plus d'un ou deux mois, sauf à disposer d'eau très froide, ou plus ou moins salée.

Lorsque l'on ne dispose que d'eau douce, à température "moyenne * (12 à $180 \mathrm{C}$ ), il peut être intéressant de stabuler ces poissons dans des bassins en 
ciment (ou en PVC), dans lesquels il est possible de les traiter preventivement ou curativement après confinement ou vidange partielle; on peut aussi, dans de tels bassins, de dimensions généralement réduites, maintenir les animaux en semi-obscurité. Dans ces conditions, certaines piscicultures obtiennent des résultats satisfaisants, mais d'autres subissent parfois des pertes importantes. surtout si les saumons ont à être conservés longtemps. Des essais infructueux réalisés à I'I.N.R.A. (St-Pée-sur-Nivelle) avant 1973 nous ont incitè à renoncer à l'utilisation de bassins de taille très réduite $\left(12 \mathrm{~m}^{2}\right)$.

Ne disposant, à la $6^{e}$ Délégation Régionale du Conseil Supérieur de la Pêche, ni d'eau salée, ni de bassins en béton adaptés à un tel mode de stabulation, tous les essais y ont été pratiqués, de 1970 à 1979, dans les bassins en terre de moyenne ou forte dimension, en esperant que le grand espace offert aux poissons pourrait être un facteur positif.

\section{TECHNIQUES DE CAPTURE}

Depuis 1970, les captures printanières ont toujours été réalisées au barrage de Vichy; c'est ce site qui, sur la rivière Allier, se prête jusquà présent le mieux à de telles opérations.

\section{A. Caractéristiques essentielles du barrage de Vichy}

Ce barrage, entièrement mobile, a été construit en 1963, pour constituer une retenue de loisirs dans la ville de Vichy.

Lorsqu'il est abaissé (généralement de fin décembre à fin mars). les saumons peuvent passer à peu près librement; le piègeage est alors impossible.

Lorsqu'il est levé, sa hauteur est de $4,50 \mathrm{~m}$. Les saumons ne peuvent alors passer que :

- par l'échelle Lachadenède, appuyée à la rive droite

- et éventuellement par le parcours canoë-kayak, contournant le barrage en rive gauche $\left({ }^{*}\right)$

$\left(^{*}\right)$ Les saumons ne s'engagent, au printemps, dans ces deux passages que lorsque la température de l'eau est supérieure à 7 ou $8^{\circ} \mathrm{C}$. Un troisième passage (échelle à bassins successifs) existe également en rive gauche : il semble ne jamais avoir été emprunté par les saumons.

Une observation importante est à faire sur le barrage de Vichy : depuis sa construction, le lit de l'Allier a été surcreusé du fait des extractions intensives de granulats; une chute d'environ un mètre (et qui s'accentue chaque année) existe donc maintenant au pied du radier de louvrage; elle risque de plus en plus de perturber la montée du saumon, surtout en périodes de faibles débits, que les vannes soient baissées ou levées; dans cette dernière position, la chute totale à franchir atteint maintenant $5,5 \mathrm{~m}$ au lieu de $4,5 \mathrm{~m}$.

Pour toutes ces raisons, des aménagements complémentaires sont de plus en plus nécessaires, afin de rendre moins aléatoire le passage des saumons au niveau de cet ouvrage. 


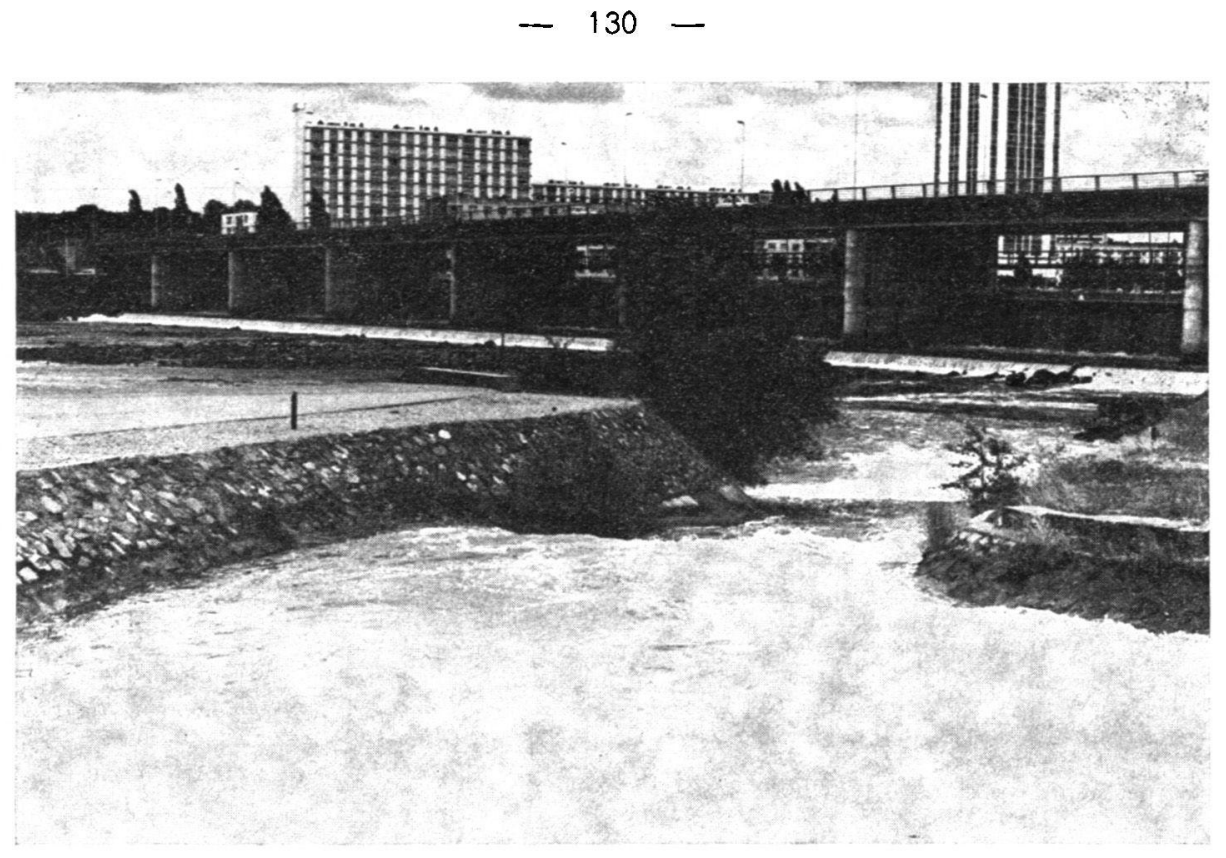

Photo 1 : Le barrage mobile de Vichy. Sur cette photo, les vannes sont levées. Au premier plan, la partie aval du parcours kayak.

\section{B. Captures dans l'échelle Lachadenède (")}

1. Les installations

C'est dans le bassin de repos amont, le plus accessible, qu'est réalisé le piégeage.

Pour cette opération. on place des grilles aux deux extrémités de ce bassin : grille verticale côté amont, interdisant toute remontée, grille oblique côté aval, rendant difficile un retour en arrière des saumons. Cette partie des installations n'a pas été modifièe depuis 1970.

Par contre, divers petits aménagements ont été apportés successivement :

- amélioration des moyens de manœuvre d'eau et d'accès au piège (1974) ;

- installation, dans la partie amont, d'un faux fond en bois, sous lequel les poissons pourraient s'abriter des turbulences (1977); le rôle de ce faux fond n'a pu être apprecié jusqu'ici ;

- installation d'un treuil desservant le bassin de repos (1978) : voir $\S 2$

(*) Passe à ralentisseurs de fond (dièdres metalliques), largeur: $2 \mathrm{~m}$. pente : $11 \%$. débit : 0.5 a $1.5 \mathrm{~m}^{3} / \mathrm{sec}$ (selon niveau amont). Dans chacun des deux bassins de repos intermediaires, la hauteur d'eau est denviron $1.5 \mathrm{~m}$. leur volume utile n'y étant que de $13 \mathrm{~m}^{3}$ environ, les turbulences y sont souvent excessives. 


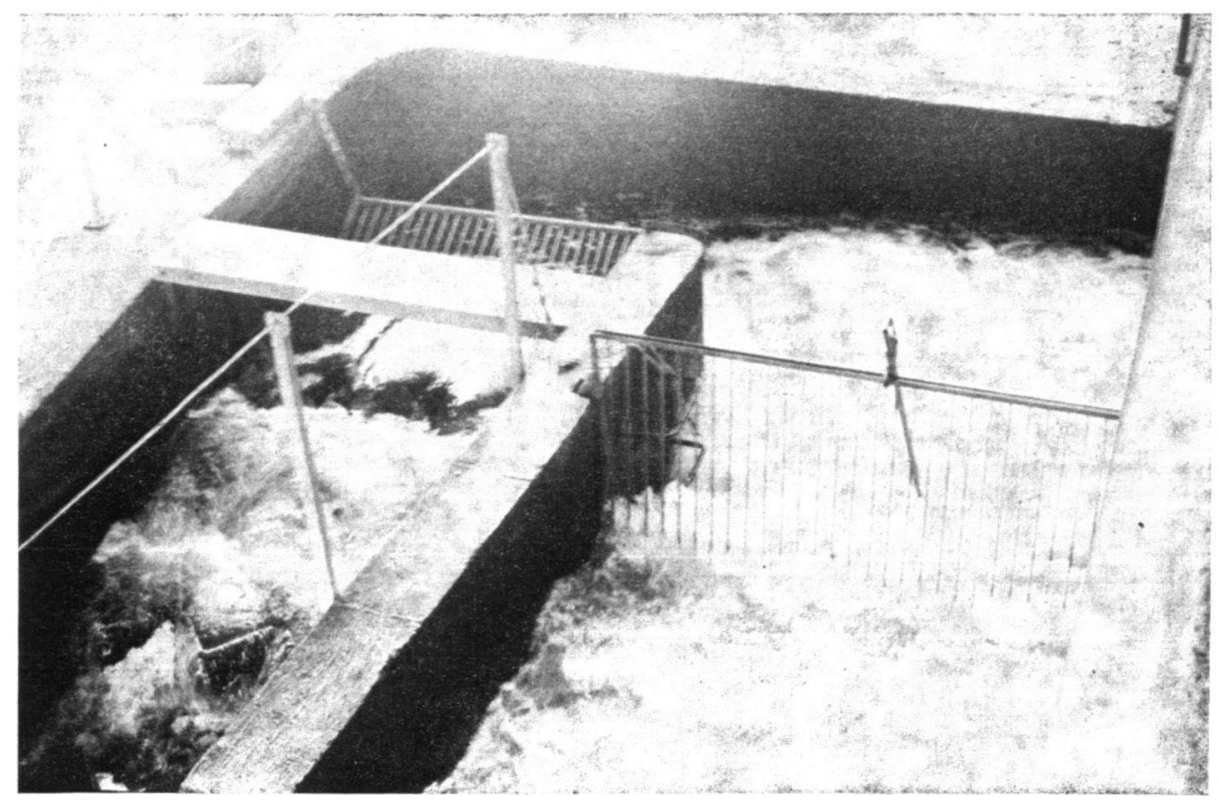

Photo 2 : Une partie de l'échelle Lachadenède, au barrage de Vichy. Les grilles de piégeage sont on place dans le bassin de repos.

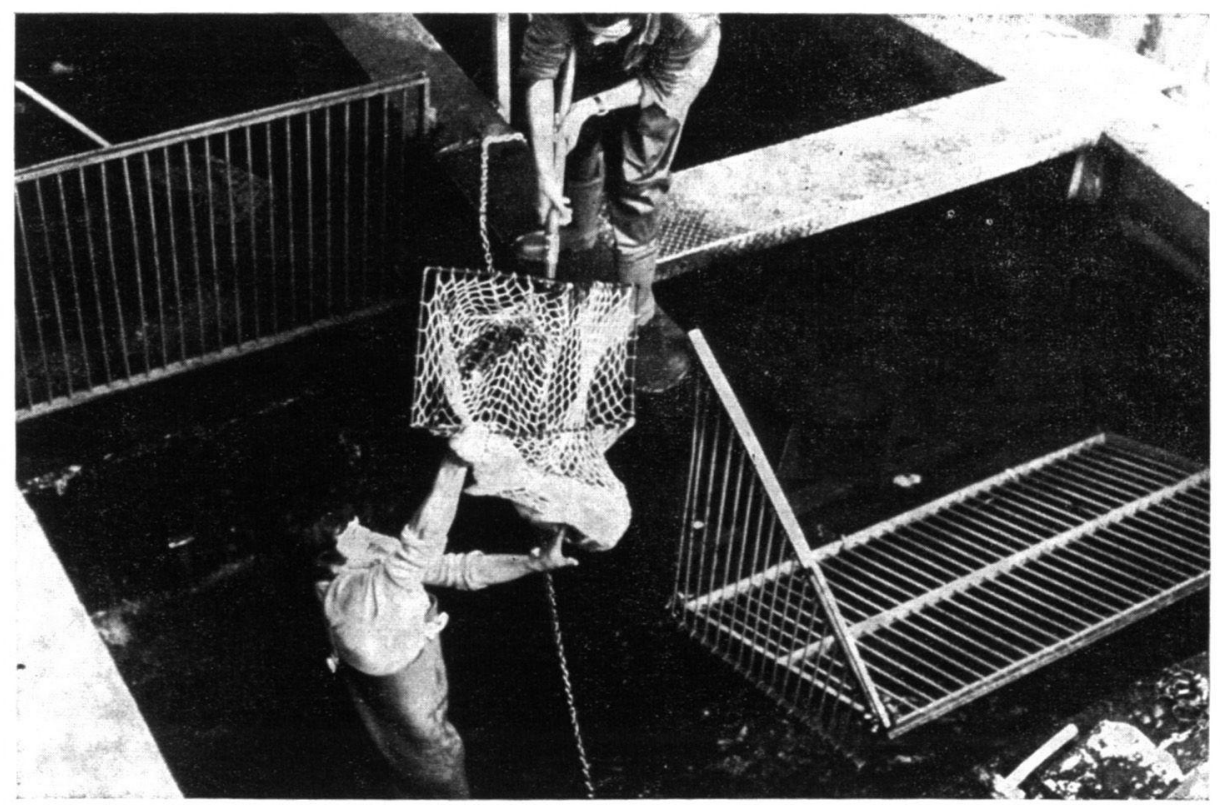

Photo 3 : Capture d'un saumon dans l'échelle Lachadenède. La chambre de repos a été partiellement vidée. Jusqu'en 1978, les saumons étaient capturés et remontés un par un dans une grande épulsette ordinaire. 


\section{Les opérations}

Pour observer ou capturer les saumons piegés dans le bassin de repos. on ferme la vanne d'admission d'eau dans l'echelle et on ouvre une vannette de vidange du bassin.

Dans les premières annees, les saumons piégés étaient retirés tous les jours ou tous les deux jours. Progressivement. on a augmenté le nombre des visites, de façon à limiter au maximum la duree de sejour de ces poissons dans le bassin de repos, où ils se fatiguent et risquent de se heurter aux parois ou grilles. Depuis 1978, le piège est visité deux à quatre fois par 24 heures si les saumons y sont assez nombreux (plus de 4 ou 5), il est pêché deux fois par jour: en aucun cas un saumon n'y est maintenu plus de 24 heures (*).

Le niveau d'eau dans le bassin de repos-piegeage est abaissè à $15-20 \mathrm{~cm}$ les saumons s'agitent peu tant que leur corps est entièrement submergè. Ils sont capturés un à un dans une epuisette à cadre rectangulaire $\left({ }^{\star \star}\right)$ et poche profonde $(40$ à $60 \mathrm{~cm})$, à grande maille $(8 \mathrm{~cm})$.

Depuis 1979 , les poches d'épuisette ont été modifiées, par adjonction d'un fond étanche. Ceci permet de maintenir chaque saumon dans l'eau pendant le levage, réalisé au moyen du treuil. Amenée au-dessus du camion de transport. la poche est videe dans le bac, sans que le poisson ait à ètre touché.

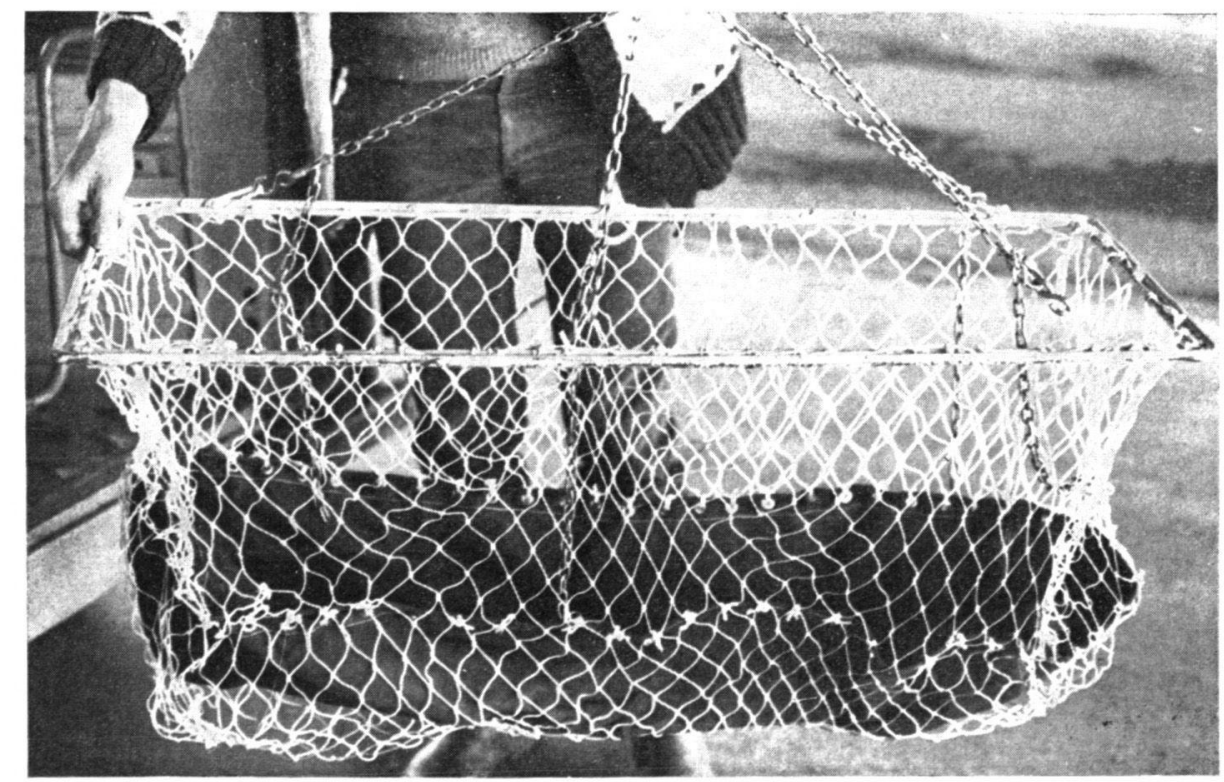

Photo 4 : Epuisette à fond étanche utilisée depuis 1979. Les saumons sont capturés et remontés un par un, dans la poche remplie d'eau, au moyen d'un bras de levage et vidés dans le bac de transport.

(*) Pour des rassons d'économie de personnel et de vehicules. Il est difficile d'auqmenter encore la fréquence des captures et des transports.

(*) Longueur : de 80 à $100 \mathrm{~cm}$ selon les cadres, largeur := $30 \mathrm{~cm}$. Le manche est amovible, pour les epuisettes utilisees avec le treul et comporte 4 anneaux d'amarrage. 


\section{Captures dans le parcours kayak (")}

Dans la période où le piège est mis en opération dans l'échelle Lachadenède. on tente également de capturer des saumons dans ce parcours, qui peut être mis partiellement à sec par fermeture de la vanne.

Cependant, l'utilisation prioritaire de cette installation par les kayaks limite les mancuvres d'eau que l'on peut y pratiquer: de nouveaux équipements y permettront ultérieurement un meilleur contrôle des montées de saumons.

Les poissons qui s'y trouvent au moment ou on y baisse le niveau (une à deux fois par jour, selon les campagnes) étaient capturés, souvent avec difficulté, à l'aide d'épuisettes normales, décrites au \& A 2. Depuis 1979, on utilise des poches en filet sans nœuds, à maille fine $(15 \mathrm{~mm})$, où les saumons sont moins susceptibles de s'abimer.

Sur les 44 sujets mis en stabulation en 1979, 19, soit $44 \%$, avaient été capturés dans le parcours kayak. Cette proportion était plus faible les années précédentes, car on pêchait moins fréguemment ce parcours

\section{Périodes et conditions de piégeage}

Les saumons passaient normalement à Vichy de décembre à avril. Depuis une dizaine d'années. ces passages sont plus tardifs, probablement du fait des nouveaux obstacles sur la Loire et l'Allier, en aval : de janvier à mai (ou même juin), avec parfois également quelques sujets "extrêmement retardataires " passant en octobre.

Les prélèvements d'adultes pour la reproduction artificielle ont été réalisés :

- généralement en avril, de 1970 à 1976

- généralement en mai, depuis 1977

Certaines années, des piégeages ont aussi èté effectués en octobre.

Chaque campagne de prélèvement a duré, selon les années, de une à quatre semaines, selon l'intensité des passages de saumons à ce moment é suivant le nombre de sujets que l'on souhaitait capturer.

Ces piégeages s'effectuent en accord avec la Fédération Départementale des A.P.P. (**) de l'Allier (dont la Briqade de Gardes-Pêche coopère généralement aux opérations), et avec l'A.P.S. ( ${ }^{* * *}$. L'autorisation administrative nécessaire est chaque fois demandée à la D.D.A. ${ }^{* * *}$ ) (Service Pêche) de ce département.

Seuls les saumons en bon état apparent sont conservés; les sujets fatigués ou blessés sont relâchés à l'amont immédiat du barrage.

On cherche aussi à prélever plus de femelles que de mâles; la distinction est malheureusement très difficile au printemps.

${ }^{*}$ ) Cette dérivation est constituée : - par un parcours kayak (écoulement rapide et diversifié) en partie aval: - par un parcours calme. utilisé partiellement par kayaks ou canoës en partie amont; le débit dans cette dérivation est commandé par une vanne. en limite entre ces deux parties; il est généralement de plusieurs $\mathrm{m}^{3} /$ seconde.

$\left({ }^{*}\right)$ A.P.P. $=$ Association agréée de Pêche et de Pisciculture

$\left({ }^{* *}\right)$ A.P.S. = Association pour la Protection du Saumon de l'Allier.

$\left.{ }^{* * *}\right)$ D.D.A. $=$ Direction Départementale de l'Agriculture. 


\section{TRANSPORT ET DEVERSEMENT A LA PISCICULTURE}

Initialement, on ne disposait que d'un petit bac métallique de 250 litros recouvert d'une planche, avec diffuseurs d'oxygène, transportés par véhicule léger.

Depuis 1974, on utilise un fourgon équipé d'une cuve en polyéthylène armé de fibre de verre, de 11001 , avec couvercle ètanche et opaque et vannes de vidange et dechargement; des diffuseurs amovibles, au fond de la cuve, sont alimentés par bouteille d'air comprimé.

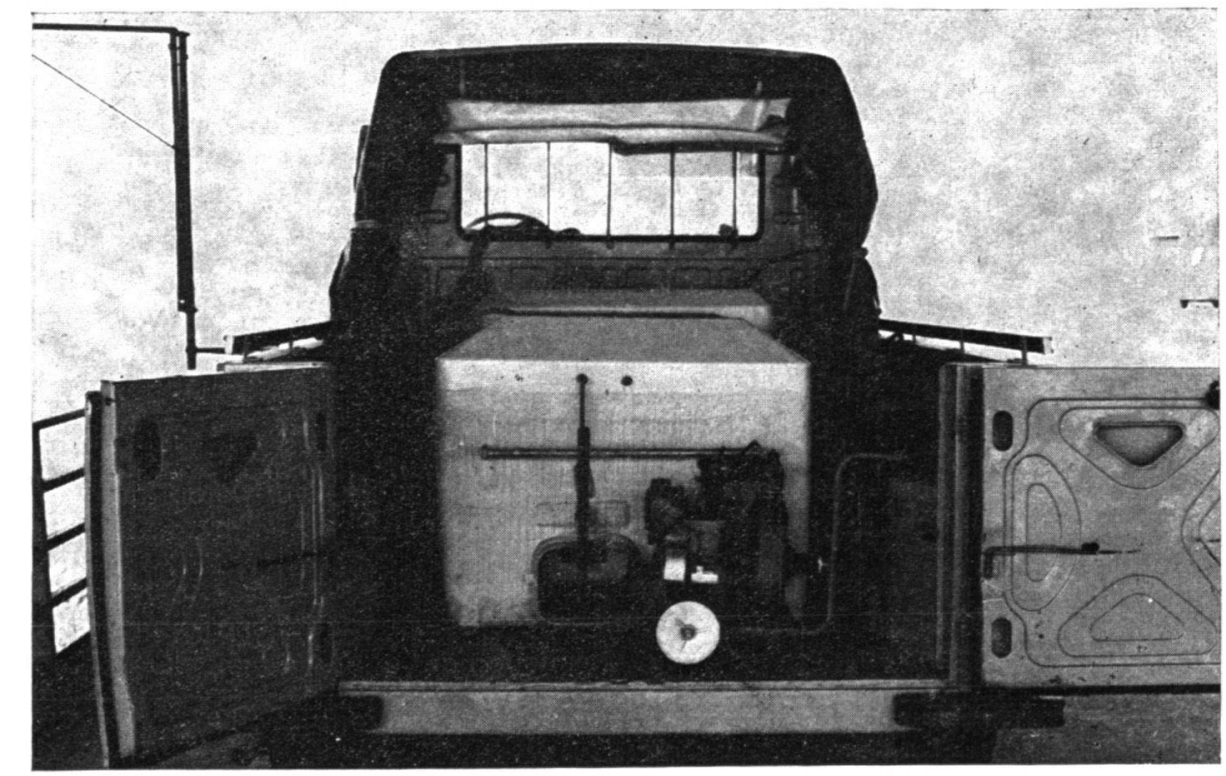

Photo 5 : Le camion de transport utilisé depuis 1979. L'absence de toit permet le chargement selon la méthode précédente (fig. 4). Au premier plan, la vanne de déchargement rapide et un groupe moto-pompe pour le remplissage. Le bras de levage est partiellement visible (à gauche).

Le transport de Vichy à la salmoniculture d'Augerolles $(60 \mathrm{~km})$ dure une heure environ; compte tenu de l'attente pendant les opérations de pêche, dans l'échelle ou le parcours kayak, des saumons restent parfois plus de deux heures dans la cuve, sans inconvénient apparent $\left(^{\star}\right)$; ils semblent toujours se tenir calmement au fond. On remplit complètement, afin de limiter au maximum le ballotement de l'eau.

On n'a jamais transporté plus de dix saumons à la fois, mais il est probablement possible d'en charger davantage. Les saumons transportés mesurent le plus souvent entre 0,75 et 1 mètre, et pèsent de 6 à $10 \mathrm{~kg}\left({ }^{\star *}\right)$.

(*) Lors de transports effectués de Vichy vers les frayères du Haut-Allier, en 1978, des saumons ont même été maintenus près de quatre heures dans la cuve, sans inconvénient apparent.

(*) Pour limiter au maximum les manipulations. les saumons ne sont jamais pesés ni mesurés lors de leur capture; leur longueur est évaluée approximativement. 


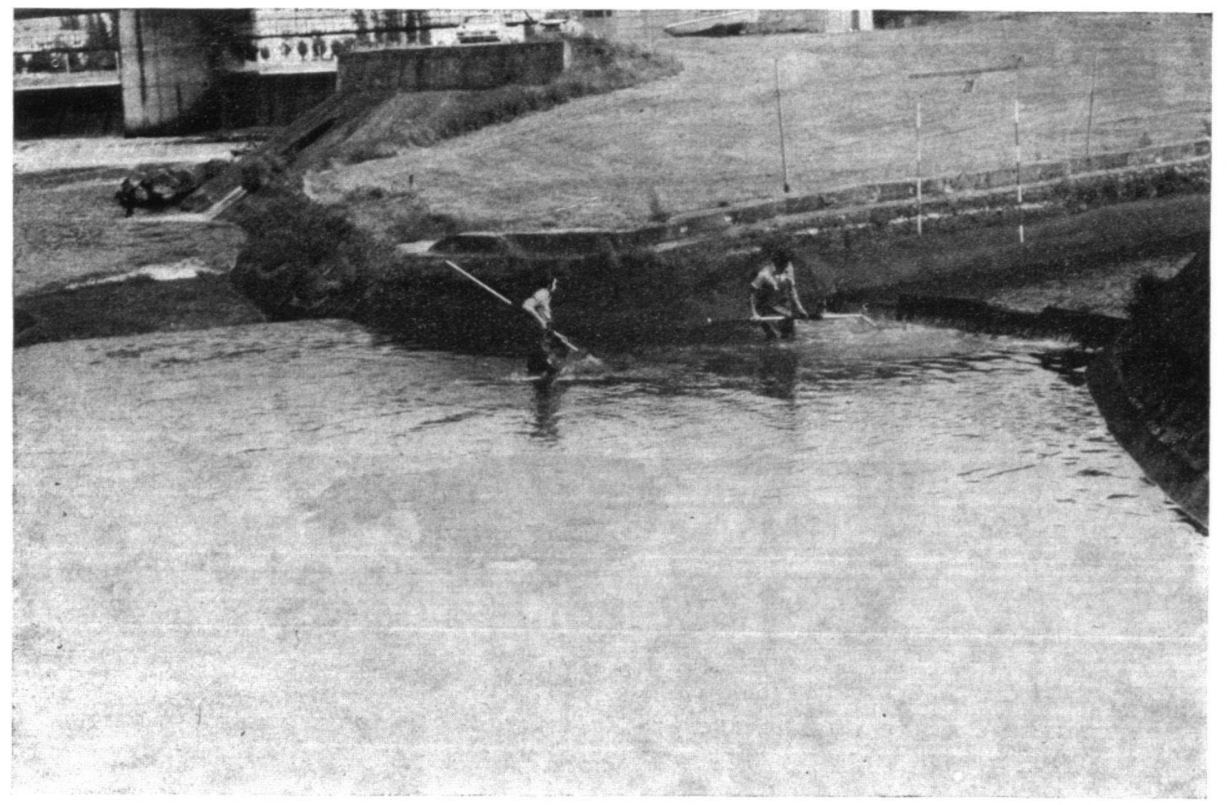

Photo 6 : Péche dans la partie aval du parcours kayak, partiellement vide. Aucun dispositif de piégeage n'existe encore dans ce parcours.

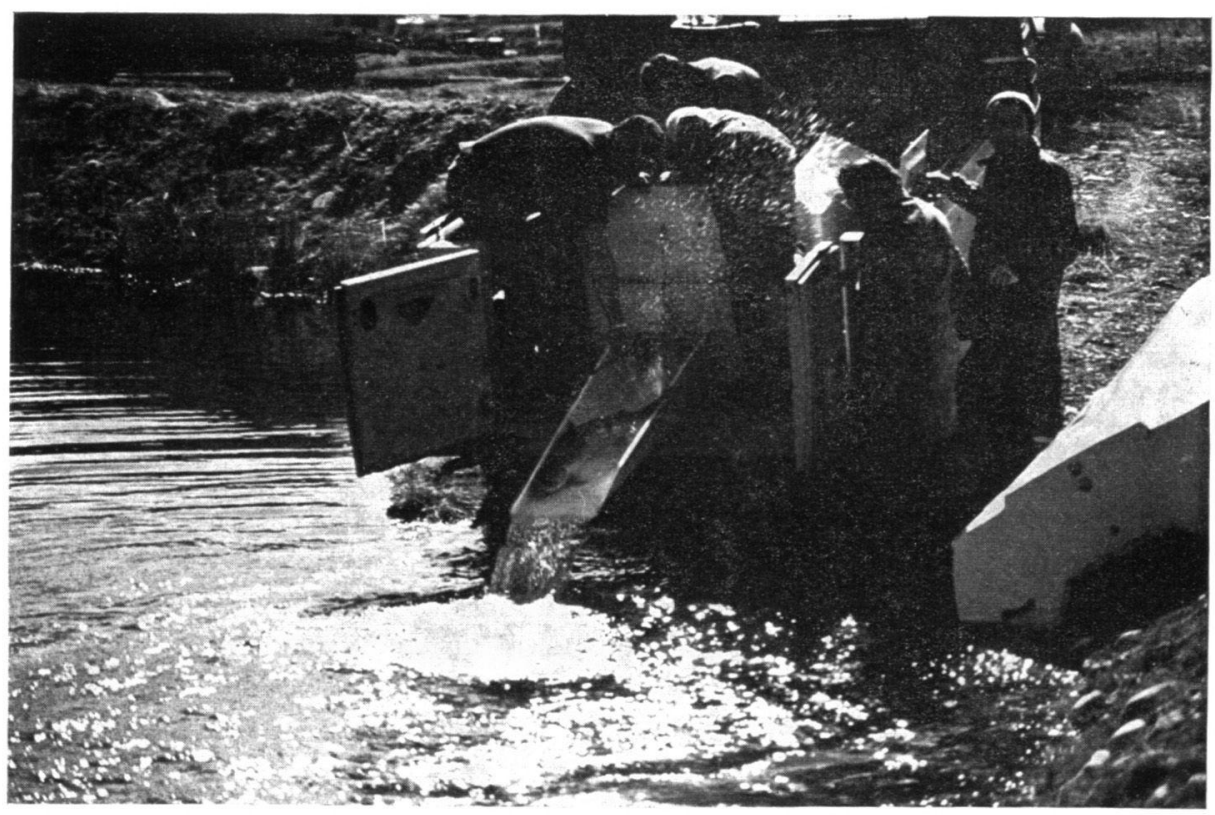

Photo 7 : Déchargement de saumons dans le bassin de stabulation d'Augerolles. Une rampe permet au camion de reculer jusqu'à l'eau. Les poissons ne sont pas manipulés lors de ce déchargement. 
Lorsque la température de l'Allier dépasse 14 à $150 \mathrm{C}$ à Vichy, on ajoute généralement un pain de glace au moment du départ, ce qui maintient ou abaisse très légerement la temperature de l'eau du bac pendant le transport

A l'arrivée à la pisciculture, les saumons sont soumis, dans la cuve

- à un equilibrage progressif des temperatures, pendant 15 à 30 minutes. au moyen du groupe motopompe et de la vidange (l'eau de la pisciculture est toujours plus froide que celle de la cuve);

- à un bain de fongicide (vert malachite) pendant trois à cinq minutes

Depuis 1977, les saumons ne sont plus sortis à l'épuisette, mais déversés directement dans le bassin de stabulation. Pour cela, on enlève les diffuseurs. on adapte une goulotte spéciale en PCV à la sortie de la vanne de déchargement, et on ouvre rapidement celle-ci : les poissons arrivent directement dans le bassin sans manipulation. Remarquons que ce système n'est possible qu'avec une cuve de grande capacite, faute de quoi l'eau s'évacue avant que certains des poissons n'aient èté entrainés.

\section{Stabulation Jusqu'a maturite SEXUELle. REsultats}

Plusieurs bassins ont successivement ete utilisés, pour stabuler les saumons du printemps jusqu'au début novembre.

\section{A. Bassins de dimensions moyennes à la salmoniculture du Conseil Supé- rieur de la Pêche à Augerolles}

De 1970 à 73 , puis en 1976, plusieurs bassins assez profonds (moyenne = $1.5 \mathrm{~m}$, maxi $=2 \mathrm{~m}$ ). mais de dimensions moyennes $\left(200\right.$ et $\left.600 \mathrm{~m}^{2}\right)$, ont été successivement employés. La temperature de "'eau allait d'environ $12^{\circ} \mathrm{C}$ au printemps à $16^{\circ} \mathrm{C}$ en èté (maxi $\cdots 18^{\circ} \mathrm{C}$ ) Le débit introduit était assez important (15 à $30 \mathrm{l} / \mathrm{sec}$ ); l'eau provenait de la Faye, affluent de la Dore (affluent de l'Allier). exempte de toute pollution.

La survie a toujours èié nuile à médiocre dans ces bassins $10 \%$ à $20 \%$ selon les années) : la plupart des sujets mouraient dans un délai de deux à six semaines apres transport, géneralement atteints de saprolegnase. Dans certains cas, des saumons ont été repris pour traitement par bains fongicides, mais sans aucun résultat : nous pensons que les stress ou chocs inèluctablement liés à de nouvelles manipulations annihilaient les effets positifs éventuels de ces traitements; il est probab'e également que la saprolégnase n'etait quiune conséquence d'autres perturbations physiologiques que nous ne savons pas diagnostiquer.

L'adjonction d'abris (buses en ciment, radeaux) n'a pas semblé améliorer la survie dans ces bassins. Des abris analogues ont également été placés dans les deux autres bassins ( $\S B$ et $C$ ), sans que nous ayons pu verifier s'ils étaient ou non frequentes par des saumons.

\section{B. Bassin de grande dimension, à Theix}

En 1973,74 et 75 , des essais ont èté pratiqués dans un bassin de $2000 \mathrm{~m}^{2}$ environ, profondeur moyenne $\cdots 1.5 \mathrm{~m}$, maxi $2.5 \mathrm{~m}$, alimenté par 10 à $20 \mathrm{l} / \mathrm{sec}$ 
d'eau de source (ayant déjà traversè 2 bassins analogues); du fait également de l'altitude $(\approx 1000 \mathrm{~m})$ et de l'ombrage important, la temperature de ce bassin restait très basse en èté (13 à $14 \circ \mathrm{C})$.

- En 1973, sur 7 saumons déversés, 5 survivants (dont deux échappés lors de la pêche), soit $71 \%$;

- en 1974, sur 35 déversés, 18 survivants $(51 \%)$, dont 9 femelles;

- en 1975, sur 42 deversés, 4 survivants seulement $(9.5 \%)$.

En 1974, et surtout 75 , le débit d'alimentation a èté sensiblement réduit (du fait de pompages dans l'aquifère). en même temps que l'eau était plus ou moins polluée par du purin. Il a fallu abandonner ce site pour ces deux raisons.

Les résultats de 1973 et 1974 constituaient déjà un très net progrès par rapport aux précédents: ils nous ont fait penser que la dimension du bassin jouait un rôle important.

\section{Bassin de grande dimension, à Augerolles}

A partir de 1977, les saumons ont été placés dans un très grand bassin $\left(\simeq 8000 \mathrm{~m}^{2}\right)$ qui venait d'être crée à la salmoniculture d'Augerolles.

La profondeur de cet "ètang" est de 2 mètres en moyenne. et de 4 mètres au maximum (près du moine).

L'eau utilisée est celle de la Faye (comme $\S A$ ), avec un débit important 150 à $200 \mathrm{l} / \mathrm{sec}$. Sa température, mesurée à 2 mètres sous la surface, ne depasse jamais $18^{\circ} \mathrm{C}$; elle est probablement légèrement inférieure à 3 ou 4 mètres de profondeur.

Elle est introduite dans le bassin par deux tuyaux débouchant au-dessous de la surface, ce qui explique probablement pourquoi les saumons sauteni peu à cet endroit; la vitesse dans ces tuyaux empêche les saumons d'y pénétrer.

En 1978 et 1979, quelques centaines d'ombres communs (Thymallus thymallus L.) reproducteurs étaient également placès dans l'étang, où on leur distribuait une nourriture artificielle d'appoint $\left({ }^{*}\right)$.

Les résultats dans ce bassin se sont très sensiblement améliorés, de 1977 à 1979 :

- en 1977, sur 14 saumons déversès 6 ( 5 et 1 ) ont survécu $(43 \%)$;

- en 1978, sur 34 déversés. 29 ont survécu (85\%); les femelles ètant encore nettement dominantes $(24 / 29)$, un bon contingent d'œufs $(220000)$ a été obtenu ;

- en 1979, sur 44 déversés, 41 ont survécu (93\%); les femelles étant moins nombreuses (23/40 sujets mâtures) et plus petites que précédemment, on prevoit d'obtenir environ $150000 œ u f s$.

Dans les premières semaines après capture, une grande partie des saumons se tiennent fréquemment rassemblès près du moine de vidange, à environ un

(*) Cette pratique ne semble avoir aucun effet défavorable aux saumons dans le méme bassin. Par contre, elle est peut-être responsable de maladies observées en 1979 dans les élevages alimentés par leau sortant de ce bassin. 


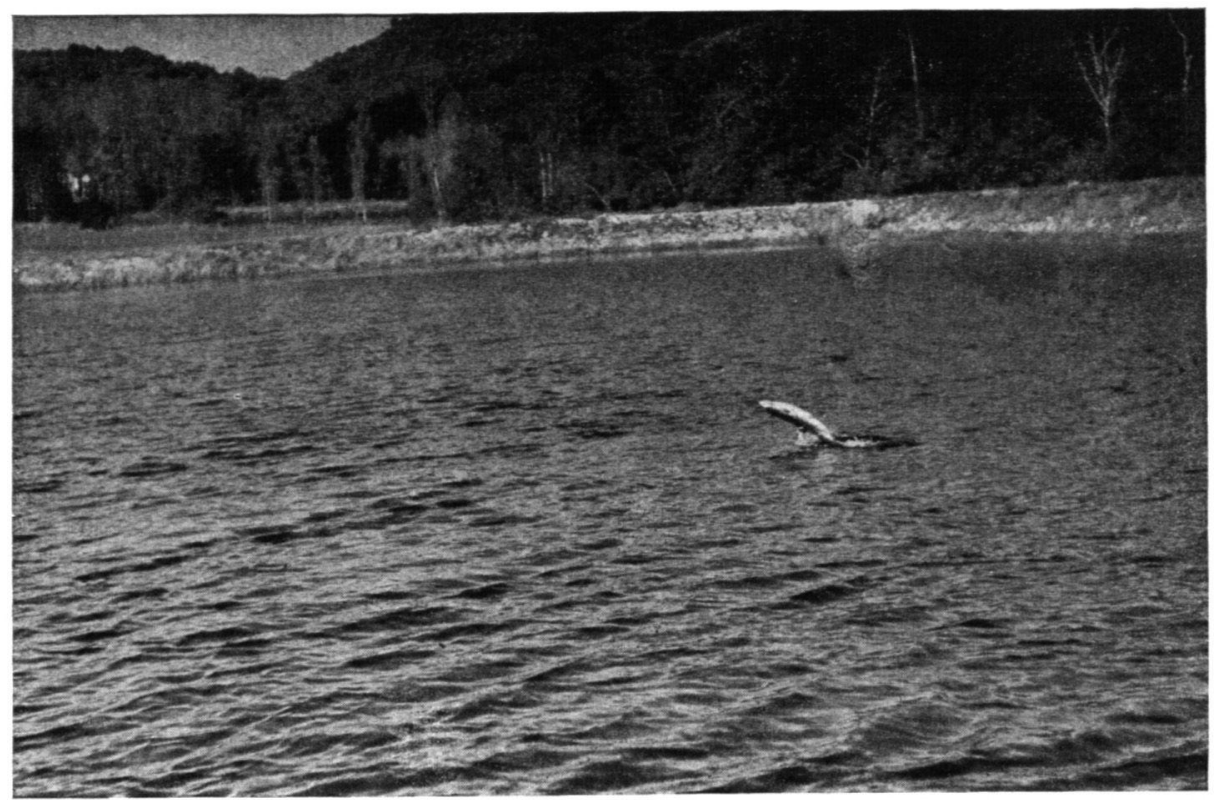

Photo 8 : Saut d'un saumon stabulé dans le très grand bassin de la salmoniculture du C.S.P. à Augerolles.

mètre sous la surface; ils semblent aussi, à d'autres moments, faire le tour du bassin. Ils paraissent plus dispersés ensuite. Il est difficile de savoir à quelle profordeur ils se tiennent. Les sauts sont fréquents, surtout en fin de journée : à l'approche de la maturité, ces sauts deviennent presque incessants $\left(^{*}\right)$.

\section{Tri des reproducteurs et ponte artificielle}

Dans tous les cas, le bassin de stabulation est vidangé entre le 1er et le 5 novembre; les saumons sont alors triés par sexe et placés dans des bassins de petites dimensions d'où on peut les reprendre facilement tous les dix jours environ pour les faire pondre, à mesure de leur maturation. Celle-ci s'échelonne normalement entre le 5 novembre et la mi-décembre.

Pour les pontes artificielles, les reproducteurs sont tranquillisés (MS 222. et plus récemment phenoxy-éthanol); depuis 1978, on emploie le dilueur de sperme mis au point par I'I.N.R.A., ce qui permet d'utiliser deux ou trois fois moins de mâles que de femelles $\left({ }^{\star \star}\right)$.

Après reproduction, les saumons sont généralement marqués (étiquette dorsale) puis transportés dans l'Allier (à Pont-du-Château ou Vichy), et même. depuis 1978, dans la Loire, près du confluent avec cette rivière, en vue d'augmenter leurs chances de deuxième remontée.

(*) Aucun saumon n'a été observé sautant sur une berge et y restant échové. Par contre. deux sujets sont morts après avoir sauté. l'un sur un a radeau. placé dans un bassin comme abri. lautre dans une barque laissée amarrée dans le très grand bassin.

(*) En cas de manque de mâles sauvages (lors des dernières pontes notamment), on utilise. sans inconvénients apparents, la laitance de juvéniles (2 ans) élevés à la pisciculture. 


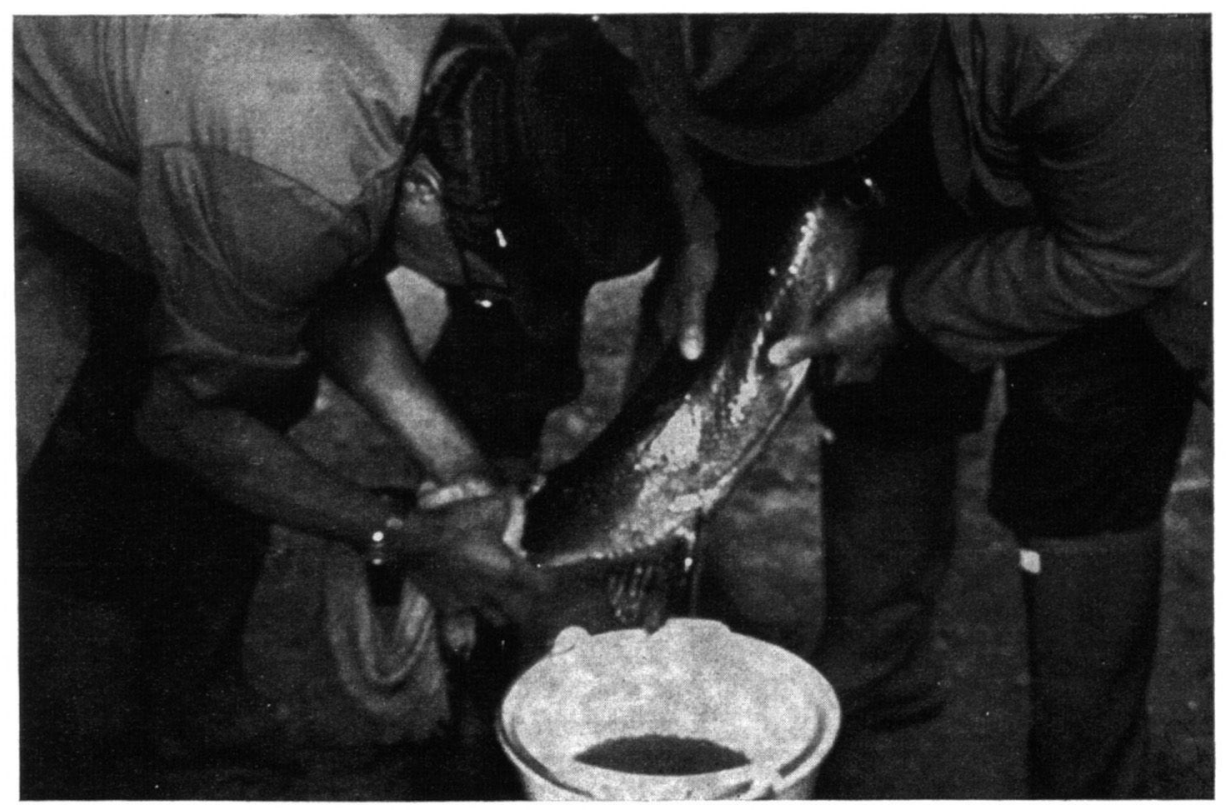

Photo 9 : Ponte artificielle d'une femelle à Augerolles. Les saumons sont repris par vidange du grand bassin au début Novembre; leur maturité s'échelonne entre le 5 et la fin Novembre (parfois jusqu'au début Décembre). lls sont endormis pour les pontes et fécondation.

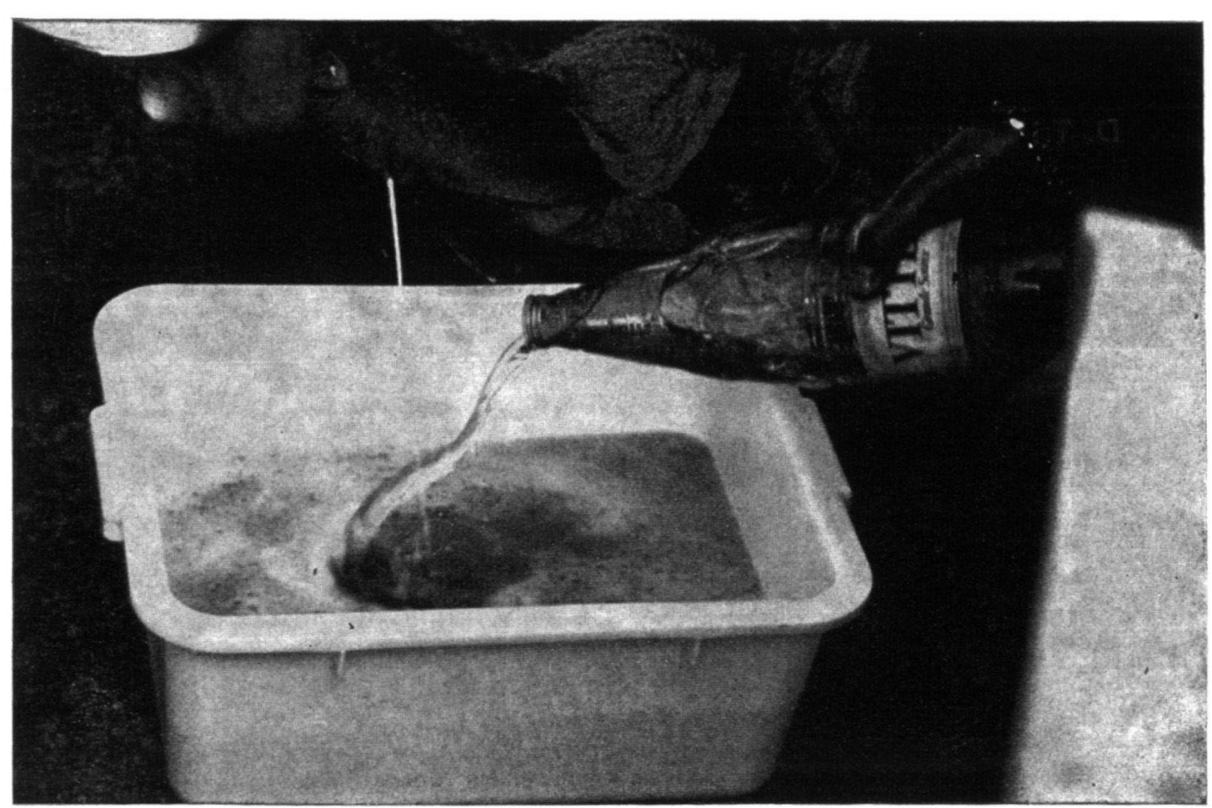

Photo 10 : Fécondation artificielle. Les ovules de plusleurs femelles ont été transférés de la passoire à la cuvette. De la laitance d'un ou deux mâles y est ajoutée. On verse aussitôt dans la cuvette un volume de dilueur égal au volume d'ovules et on mélange. 


\section{DISCUSSION ET CONCLUSIONS}

Le nombre de saumons mis en stabulation au printemps a beaucoup varie selon les années, de 1970 à 1976 : de quelques unités à 44 au maximum.

\section{Les survies obtenues ont èté :}

a) nulles ou infimes dans les bassins de taille *moyenne " à Augerolles $(1970,71,72,73,76)$, malgré l'alimentation en fort débit d'eau de très bonne qualité ;

b) moyennes $(55 \%)$, puis faible $(9 \%$ ) après pollution, dans le grand bassin de Theix, à temperature basse

c) très bonnes $(83,7 \%$ en moyenne) dans le très grand bassin à Augerolles, pourtant alimenté exactement par la même eau que dans les opérations (a), ce qui coinfirme l'importance de la dimension du bassin: l'amélioration progressive constatée dans ce mème bassin, de 1977 à $79(43 \%, 85 \%, 93 \%)$, résulte très probablement des améliorations apportées, dans cette periode, aux équipements et techniques de capture et transport

La taille du bassin apparait bien comme un facteur determinant dans la survie de saumons stabules pendant une longue pèriode: les effets psychosomatiques défavorables liés au confinement et à la captivité sont certainement particulièrement marqués chez cette espéce

La forte profondeur permet à ces poissons, non seulement de se sentir probablement plus en securité, mais aussi de disposer d'un certain choix quant à la temperature. On sait que, dans l'Allier, le saumon estive dans des fosses profondes, ou il peut trouver à la fois des abris ei des temperatures moins éevees que celles de l'eau "circulante " de la rivière (atteignant parfois 23 " $\mathrm{C}$ ).

11 est difficile de savoir combien de sujets on pourrait stabuler au maximum dans un tel bassin : la denslié de 1979 ètait de 55 saumons par hectare (chacun disjosait de $180 \mathrm{~m}^{2}$ en moyenne) : pour un poids individue! moyen apprnximatif de $6 \mathrm{~kg} \mathrm{(").} \mathrm{la} \mathrm{biomasse} \mathrm{correspondante} \mathrm{est} \mathrm{d'environ} 330 \mathrm{~kg} / \mathrm{ha}$. Ces valeurs paraissent faibles. Rappelons cependant que les saurnons, souvent rassemblés, ne semblent utiliser, surtout au début. qu'une faible partie de la surface du bassin; il nous semblerait imprudent de désasser dans ces conditions une charge de 450 à $500 \mathrm{~kg} / \mathrm{ha}$ (so:t, pour des sujets de $7 \mathrm{~kg}$ en moyenne. 55 à 60 dans ce bassin).

$$
*
$$

Mais les conditions de capture, transport et manipulations ont aussi une grande importance. L'amélioration des résultats, dans le même bassin, de 1977 à 1979, en mème temps que le matériel et les précautions utilisés pour ces opérations se sont perfectionnes, en est un très bon indice. Le personnel de la 6 e

(*) Poids approximatif lors des captures. au printemps: il est réduit de 20 a $30 \%$ lorsque ces poissons atteignent leur maturité sexuelle. 
Délégation Régionale du Conseil Supérieur de la Pêche, et plus particulièrement les Gardes-Pêche de la "Brigade Mobile de Surveillance" et de la "Camion. nette Technique", ont acquis une bonne expérience pratique de l'ensemble des opérations et apportent un soin maximum à toutes les manipulations.

Une autre preuve semble avoir été donnée a contrario lors du transfert dans le mème bassin, en avril 1979, de huit saumons capturés au filet en Loire moyenne : capturés, stockés en viviers (certains plusieurs jours) et transportés dans des conditions à notre avis defectueuses, ils sont tous morts en moins d'un mois et demi $\left(^{*}\right)$.

Tous ceux qui ont tenté de conserver longtemps des saumons reproducteurs connaissent l'extrême fragilité de ce poisson : s'il a subi des traumatismes ou des stress excessifs lors de la capture, du transport, ou de manipulations diverses, ses chances de survie deviennent très faibles, même si aucun symptôme n'est encore apparent dans les premiers jours.

Quelques améliorations de détail pourront encore être apportées aux techniques et au matériel, dans les prochaines campagnes, de façon à faciliter encore les opérations. L'installation. dans le parcours canoé-kayak, d'un piège temporaire serait notamment très souhaitable.

Mais c'est plutôt à deux autres niveaux que des améliorations seraient maintenant intéressantes, en vue d'augmenter le nombre d'œufs, puis de tacons pour le repeuplement, à partir du plus faible prélèvement possible d'adultes. II serait utile, principalement

- de disposer d'un moyen de reconnaitre le sexe des saumons dès le printemps, afin de ne prélever que le minimum de mâles nécessaires aux fécondations, pour en laisser un maximum pour les captures des pêcheurs ;

- et surtout d'améliorer la survie, encore insuffisante à la salmoniculture d'Augerolles (15 à $25 \%$ selon les années), de l'œuf jusqu'au saumoneau (smolt) libéré dans l'Allier.

Au point de vue génétique, il est probable que le fait de toujours utiliser pour les repeuplements des sujets plutôt tardifs constitue une sélection défavarable, à terme. Pour corriger cette tendance, on tentera de prélever quelques mâles à migration hâtive et de les utiliser pour un maximum de fécondations. Ce procédé se heurte cependant à de sérieuses difficultés pratiques.

\section{$* *$}

La possibilité, qui semble maintenant acquise pour l'Allier, de mieux maitriser l'abtention d'œufs permet maintenant d'envisager plus sérieusement la création d'une deuxième salmoniculture, plus importante que celle d'Augerolles: ceci afin d'intensifier les repeuplements dont le Saumon Loire-Allier n'a jamais eu autant besoin que dans la periode actuelle.

(*) On doit noter cependant que ces saumons se trouvaient en rivière depuis moins lonqtemps que ceux que nous capturons en mai à Vichy et que la * fragilité * de ces poissons (notamment le risque de perte d'écailles) semble diminuer à mesure que le temps passé en eau douce augmente. 